\title{
An optimized registration workflow and standard geometric space for small animal brain imaging
}

\author{
Journal Article \\ Author(s): \\ loanas, Horea-loan (D); Marks, Markus; Zerbi, Valerio (D); Yanik, Mehmet Fatih (D); Rudin, Markus \\ Publication date: \\ 2021-11-01 \\ Permanent link: \\ https://doi.org/10.3929/ethz-b-000499571
}

Rights / license:

Creative Commons Attribution 4.0 International

Originally published in:

Neurolmage 241, https://doi.org/10.1016/j.neuroimage.2021.118386 


\title{
An optimized registration workflow and standard geometric space for small animal brain imaging
}

\author{
Horea-Ioan Ioanas ${ }^{\mathrm{a}, *}$, Markus Marks ${ }^{\mathrm{b}, \mathrm{d}}$, Valerio Zerbi ${ }^{\mathrm{c}}$, Mehmet Fatih Yanik $^{\mathrm{b}, \mathrm{d}}$, Markus Rudin ${ }^{\mathrm{a}}$ \\ a Institute for Biomedical Engineering, ETH and University of Zurich, Switzerland \\ ${ }^{\mathrm{b}}$ Institute of Neuroinformatics, D-ITET, ETH and University of Zurich, Switzerland

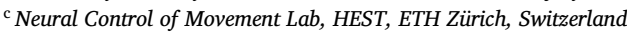 \\ ${ }^{\mathrm{d}}$ Neuroscience Center, ETH and University of Zurich, Switzerland
}

\section{A B S T R A C T}

The reliability of scientific results critically depends on reproducible and transparent data processing. Cross-subject and cross-study comparability of imaging data in general, and magnetic resonance imaging (MRI) data in particular, is contingent on the quality of registration to a standard reference space. In small animal MRI this is not adequately provided by currently used processing workflows, which utilize high-level scripts optimized for human data, and adapt animal data to fit the scripts, rather than vice-versa. In this fully reproducible article we showcase a generic workflow optimized for the mouse brain, alongside a standard reference space suited to harmonize data between analysis and operation. We introduce four separate metrics for automated quality control (QC), and a visualization method to aid operator inspection. Benchmarking this workflow against common legacy practices reveals that it performs more consistently, better preserves variance across subjects while minimizing variance across sessions, and improves both volume and smoothness conservation RMSE approximately 2-fold. We propose this open source workflow and the QC metrics as a new standard for small animal MRI registration, ensuring workflow robustness, data comparability, and region assignment validity, all of which are indispensable prerequisites for the comparability of scientific results across experiments and centers.

\section{Background}

Correspondence of brain areas across individuals is a prerequisite of generalizable statements regarding brain function and organization. This is achieved by spatial transformation of brain maps in a study to a population or study reference template. This process, called registration, is integral to any neuroimaging workflow attempting to produce results which are both spatially resolved and meaningful at the population level.

The computations required for registration are commonly performed in the preprocessing workflow, though image manipulation may only take place once inter-subject comparison becomes needed. As a consequence of this peripheral positioning in the data evaluation sequence, and of the independence from experimental designs and hypotheses, registration is often relegated to default values and exempt from rigorous design and quantitative QC efforts.

Registration in human brain imaging benefits from highlevel functions (e.g. flirt and fnirt from the FSL package Jenkinson et al. (2012), or antsIntroduction.sh from the ANTs package Avants et al. (2011)), optimized for the size and spatial features of the human brain. In small animal brain imaging, registration is performed via the selfsame high-level functions as human brain imaging - rendered usable by adjusting the data to fit function parameters, rather than vice-versa. This approach compromises data veracity, limits optimization potential, and represents a notable hurdle for the methodological improvement of small animal brain imaging.

Ad-hoc workflows for preclinical imaging often emerge as the need mandates, but lack broad reusability due to highly variable protocols for data acquisition across centers Carp (2012). While further advancements are being made for general-purpose human data preprocessing Esteban et al. (2019), the preclinical field still lacks similarly reliable tools. Recently, a preclinical registration framework has been released Anderson et al. (2019), dealing specifically with structural recordings focused on voxel-based morphometry (VBM) and validated via phantombased metrics. General-purpose registration workflows applicable both to functional and structural data remain lacking, as do benchmarking efforts which take into account QC metric challenges arising from the heterogeneity of in vivo experiments.

Below, we explicitly describe current practices used in extant ad-hoc workflows, in an effort to not only propose better solutions, but do so in a falsifiable manner which provides adequate detail for both novel and the legacy methods.

\subsection{Manipulations}

The foremost data manipulation procedure in present-day small animal MRI is scale adjustment. Scale is represented by the Neuroimaging Informatics Technology Initiative format (NIfTI) Cox et al. (2004) affine

\footnotetext{
* Corresponding author.

E-mail addresses: horea@mit.edu, chr@chymera.eu (H.-I. Ioanas).
} 
transformation parameters, which map the data matrix to spatial coordinates. Commonly, this manipulation constitutes a 10-fold increase in each spatial dimension.

In order to produce acceptable results from brain extraction based on human priors, it may be necessary to additionally adjust the data matrix content itself. This may involve applying an ad-hoc intensitybased percentile threshold to delete non-brain as well as anterior and posterior brain voxels, and leave a more spherical brain for human masking functions to operate on. While conceptually superior solutions adapting parameters to animal data are available Oguz et al. (2014); Wood et al. (2013) and might remove the need for this step of data adaptation, rudimentary solutions remain popular. Both these function adaptations for animal data and the animal data matrix content adaptations for use with human brain extraction functions are, however, prone to completely or partially remove the olfactory bulbs. For this reason, the choice is sometimes made to simply forego brain extraction.

Scan orientation metadata may be seen as problematic, and consequently deleted. This consists in resetting the NIfTI S-Form affine to zeroes, and is intended to mitigate orientations incompatible with the target template. While it is true that scanner-reported affine spaces for small animal data may be nonstandard (the confusion is two-fold: small animals lie prone with the coronal plane progressing axially whereas higher primates lie supine with the horizontal plane progressing axially), affine spaces of small animal brain templates may be nonstandard as well. A related manipulation is dimension swapping, which changes the order of the NIfTI data matrix dimensions rather than the affine metadata. Thus, correct or automatically redressible affine parameters can be deleted and data degraded beyond easy recovery, in order to correspond to a malformed template.

\subsection{Templates}

As the above demonstrates, the template is a key dependency for registration. Templates used for small animal brain MRI registration are heterogeneous and include histological as well as ex vivo MRI templates, scanned either inside the intact skull or after physical brain extraction.

Histological templates benefit from high spatial resolution and, in the mouse model in particular, access to molecular information in the same coordinate space. Such templates are not produced in volumetric sampling analogous to MRI, are hard to register to due to their contrast, may be severely deformed due to their extraction process, and are often not assigned a meaningful affine transformation after conversion to NIfTI. Data registered to templates thus deformed is challenging to use for navigating the intact brain during stereotactic surgery.

Ex vivo templates based on extracted brains share most of the deformation issues present in histological templates; they are, however, available in MR contrasts, making registration more reliable. Ex vivo templates based on intact heads provide both MR contrast and brains largely free of deformation and supporting whole brain registration.

\subsection{Challenges}

The foremost challenges in small animal MRI registration consist in eliminating data-degrading workarounds, reducing reliance on highlevel interfaces with inappropriate optimizations, and improving the quality of standard space templates. Information loss (pertaining to both the affine and the data matrix) is a particularly besetting issue, since the loss of data at the onset of a neuroimaging workflow persists throughout downstream steps and precludes numerous modes of analysis (fig. $1 \mathrm{a}$ vs. fig. 1b). Additionally, given data heterogeneity in various MRI applications, processing effects need to be evaluated with respect to at least the most common contrasts, such as anatomical $T_{1}$ and $T_{2}$-weighted imaging, as well as variations of functional contrast, such as cerebral blood volume (CBV) and blood-oxygen-level-dependent (BOLD) imaging.

\section{The optimized workflow}

Processing workflow complexity should be manageable to prospective users with only cursory programming experience. However, workflow transparency, sustainability, and reproducibility should not be compromised for trivial ancillary features. We thus abide by the following design guidelines: (1) each workflow is represented by a high-level function, with operator-understandable parameters, detailing operations performed, rather than implementations; (2) workflow functions are highly parameterized but include workable defaults, so users may significantly adapt workflows without editing constituent code; (3) graphical or interactive interfaces are avoided, as they impede reproducibility, encumber the dependency graph, and reduce project sustainability.

Given the aforementioned principles, we have constructed two registration workflows: The "Legacy" workflow (fig. 1c, exhibiting the common practices detailed in the Background section) and the novel "Generic" workflow (fig. 1d), Both workflows start by performing dummy scan correction on the fMRI data and the stimulation events file, based on BIDS Gorgolewski et al. (2016) metadata, if available. The "Legacy" workflow subsequently applies a 10-fold multiplication to the voxel size, and deletes the orientation information from the affine matrix. Further, dimensions are swapped so that the data matrix matches the RPS (left $\rightarrow$ Right, anterior $\rightarrow$ Posterior, inferior $\rightarrow$ Superior) orientation of the corresponding template (fig. 2b). Following these data manipulation steps, a temporal mean is computed, and an empirically determined threshold ( $10 \%$ of the $98^{\text {th }}$ percentile) is applied. Subsequently, the bias field is corrected and masked using the fast and bet FSL functions. The image is then warped into the template space using the antsIntroduction.sh ANTs function. Lastly, affine variants are harmonized. The "Generic" workflow follows up on dummy scan correction with slice timing correction, computes the temporal mean of the functional scan, and bias corrects the temporal mean - using the N4BiasFieldCorrection ANTs function, with spatial parameters adapted to small animal brain features. Analogous operations are performed on the structural scan, following which the structural scan is registered to the reference template, and the functional scan temporal mean is registered to the structural scan - using the antsRegistration ANTs function, with spatial parameters adapted to small animal brain features. The structural-to-template and functional-to-structural transformation matrices are merged and applied in one warp step to the functional data, while the structural data is warped solely via the structuralto-template transformation matrix.

General-purpose small animal support is provided in the node optimizations via scale reduction across all parameters, as well as iteration reduction for registration nodes. The common features of preclinical models thus supported are the smaller scale, smoother brain surfaces, and potential exclusion of the caudalmost and rostralmost areas of the brain.

The workflow is implemented in Python, with automatically generated Command Line Interfaces (CLIs) available for Bash. As registration is indispensable to data analysis (rather than a stand-alone process), the workflows are distributed as part of a comprehensive workflow package, SAMRI Ioanas et al. (2017a).

\subsection{Template package}

Target template quality is highly consequential to registration performance and suitability as a standard procedure. For example, an inflated template size mandates according parameters for all functions handling data in affine space. Further, if template axes are flipped, the first (rigid) registration step may be incorrectly determined and the image deformed to match the template at an incorrect orientation. Consequently, template quality must be ascertained, and a workflow-compliant default should be provided.

Our recommended template (fig. 2a) is derived from the DSURQE template of the Toronto Hospital for Sick Children Mouse Imaging Cen- 


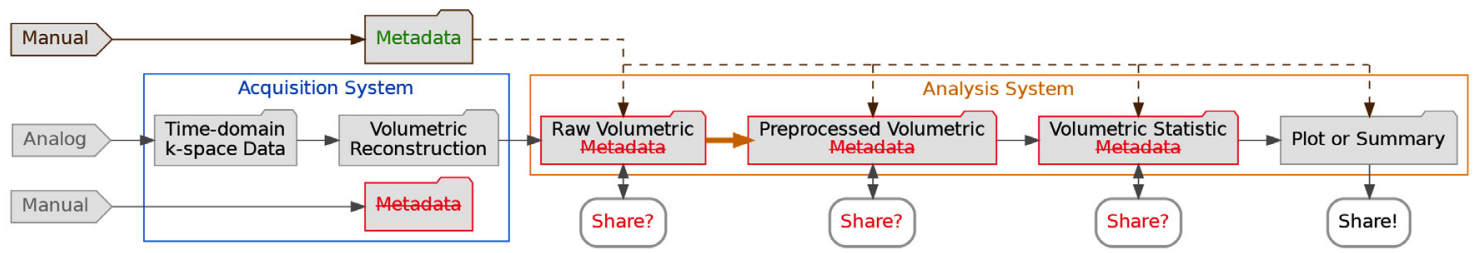

(a) Ad hoc metadata substitution irreversibly damages data and hinders reuse and sharing at all downstream levels.

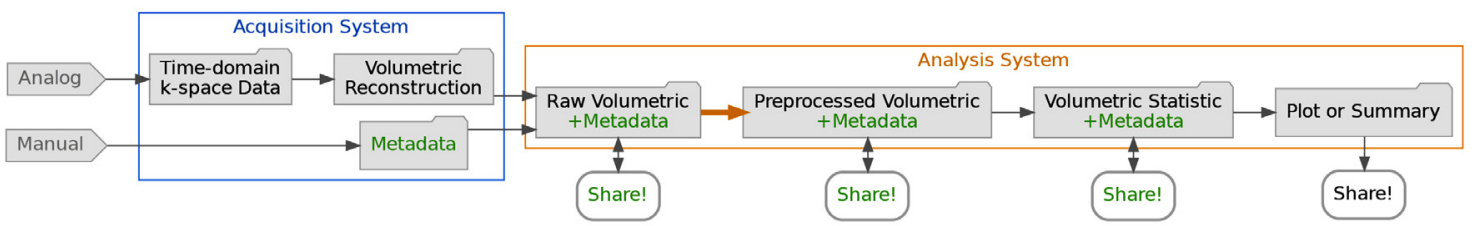

(b) Nondestructive handling of metadata ensures reusability and easy sharing throughout the analysis process.

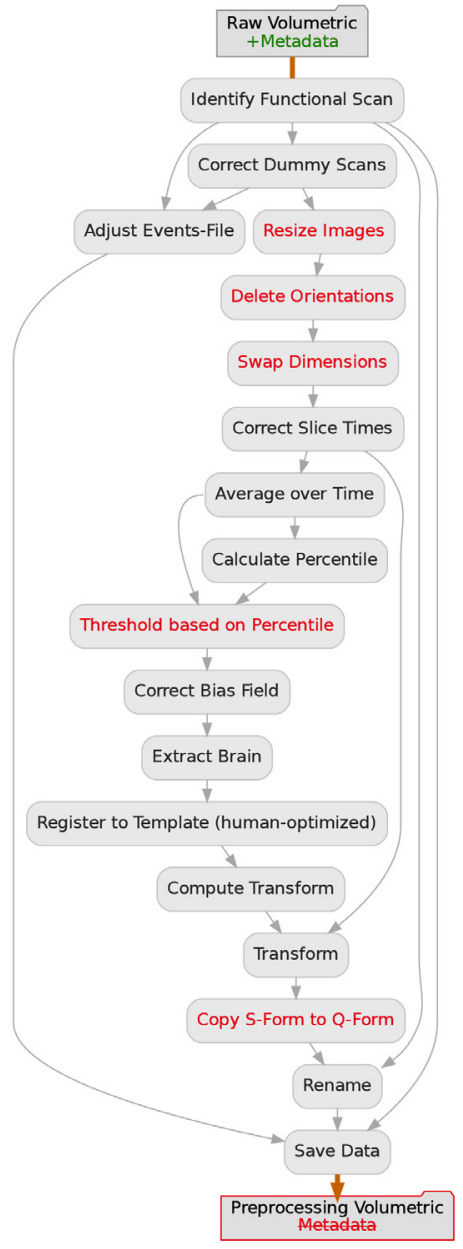

(c) "SAMRI Legacy" workflow, based on the antsintroduction. sh function, and including destructive manipulations in nodes colored red.

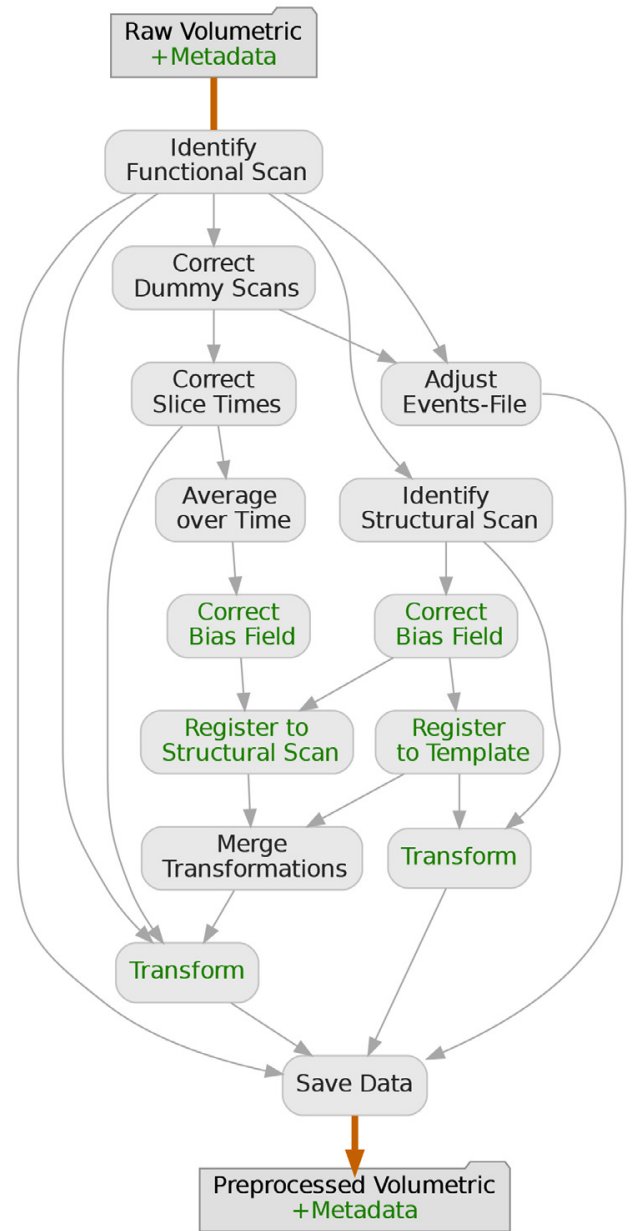

(d) Non-destructive "SAMRI Generic" workflow, based on the antsRegistration function, and including small-animal-specific parameter optimization in nodes colored green.

Fig. 1. The SAMRI Generic workflow uses fine-tuned animal priors to enhance registration quality while preserving metadata integrity. Directed graphs depict both the overall context of MRI data processing and analysis (a,b), as well as the internal structure of the two registration workflows compared in this article (c,d), which insert into the broader context at the bold orange arrow positions. Technical detail available in fig. S5.

ter Dorr et al. (2008). We shift its geometric origin to the Bregma landmark, providing harmonization with histological atlases and surgical procedures. The template is in the canonical NIfTI orientation, RAS (left $\rightarrow$ Right, posterior $\rightarrow$ Anterior, inferior $\rightarrow$ Superior), and has a coronal slice positioning typical for both MRI and stereotactic surgery. All of the associated files are identified with the prefix dsurqec in the template package.

We bundle the aforementioned MR template with histological templates, derived from the Australian Mouse Brain Mapping Consortium (AMBMC) Janke and Ullmann (2015) and Allen Brain Institute (ABI) 

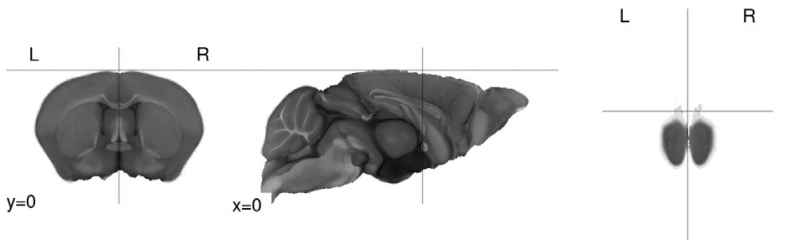

$z=0$

(a) The "Generic" template: MR and stereotactic data matrix orientation, standard header with RAS orientation and conserved scale, and histologically meaningful Bregma origin.
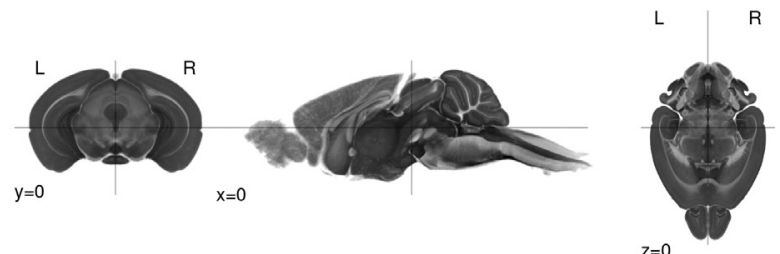

(b) The "Legacy" template: histological data matrix orientation (shared e.g. by the Allen Brain Institute template), and nonstandard header with RPS orientation and inflated scale.

Fig. 2. The "Generic" template provides canonical orientation and Bregma centering. Illustrated are multiplanar depictions of the "Generic" and "Legacy" $T_{2}$ contrast mouse brain templates, with slice coordinates centered at zero on all axes.

Lein et al. (2006) templates, which provide ample rostrocaudal coverage and serve as a reference for numerous gene expression and projection maps, respectively. We reorient the AMBMC template from its original RPS orientation to the canonical RAS, and apply an RAS orientation to the orientation-less ABI template after NIfTI conversion from the original NRRD format. Corresponding files are prefixed with ambmc and abi, respectively.

Additionally, we provide DSURQEC and AMBMC derived templates, in the widespread but incorrect RPS orientation, with the aforementioned tenfold increase in voxel size. These are prefixed with ldsurqec and lambmc, respectively.

All templates are provided at $40 \mu \mathrm{m}$ and $200 \mu \mathrm{m}$ isotropic resolutions. Up-to-date versions of these archives can be reproduced via a script collection written and released for the purpose of this publication Ioanas et al. (2019b).

For evaluation, dsurqec and ldsurqec template variations (same data matrix, matched to the orientation and size requirements of the fig. $1 \mathrm{~d}$ and fig. 1c workflow functions, respectively) are referred to as the "Generic" template. Analogously, the ambmc and lambmc template variations are referred to as the "Legacy" template.

\subsection{Qualitative operator inspection}

We complement automated whole-dataset evaluation metrics with convenience functions to ease and improve qualitative inspection. These functions produce paginated slice-by-slice views of registered data, highlighting two different assessments. The first view mode highlights singlesession registration quality, plotting the registered data as a map, and the target atlas as a contour (figs. S1 and S2). The second highlights multi-session registration coherence, plotting the target template as a map, and colored individual session contours (fig. S3).

\section{Evaluation}

A major challenge of registration QC is that a perfect mapping from the image to the template is undefined. Similarity metrics are ill-suited for QC, as they are used internally by registration functions, whose mode of operation is based on optimizing them. Moreover, similarity metrics are not independent, so similarity score optimization issues cannot be circumvented by selecting a subset of metrics and performing QC via the remainder. To address this challenge, we developed four alternative evaluation metrics: volume conservation, smoothness conservation, functional analysis, and variance analysis. In order to mitigate possible differences arising from template features, we use these metrics for multi-factorial analyses - including both a template and a workflow factor. In order to evaluate robustness with respect to data contrast, we additionally present a multi-factorial break-down of metric variability given contrast combinations of the functional data (BOLD and CBV) and associated structural data (T1-weighted FLASH and T2-weighted RARE).

\subsection{Volume conservation}

Volume conservation is based on the assumption that brain volume should remain roughly constant after preprocessing. Assuming size consistency between data and template, a volume increase may indicate that the brain was stretched to fill template space not covered by the scan, while a volume decrease might indicate that non-brain voxels were introduced into the template brain space. For this analysis we compute a Volume Conservation Factor (VCF), whereby volume conservation is optimal for $\mathrm{VCF}=1$.

As seen in fig. 3a (left panel), we note that VCF is sensitive to the workflow $\left(F_{1,388}=182.8, p=2.12 \times 10^{-34}\right)$, the template $\left(F_{1,388}=\right.$ $\left.569.3, p=4.28 \times 10^{-78}\right)$, but not the interaction thereof $\left(F_{1,388}=0.56, p=\right.$ .45). The performance of the Generic SAMRI workflow in conjunction with the Generic template is significantly different from that of the Legacy workflow in conjunction with the Legacy template, yielding a two-tailed p-value of $1.1 \times 10^{-20}$. Moreover, the root mean squared error ratio strongly favours the Generic workflow $\left(\operatorname{RMSE}_{\mathrm{L}} / \mathrm{RMSE}_{\mathrm{G}} \simeq 2.7\right)$.

Descriptively, we observe that the Legacy level of the template variable introduces a notable volume loss (VCF of $-0.32,95 \% \mathrm{CI}:-0.34$ to 0.30 ), while the Legacy level of the workflow variable introduces a volume gain (VCF of $0.18,95 \% \mathrm{CI}: 0.16$ to0.20). Further, we note that there is a very strong variance increase in all applications of the Legacy workflow - 5.9-fold (variances of 0.013 and $2 \times 10^{-3}$ for the Legacy and Generic workflows, respectively) given the Legacy template, and 3.9fold (variances of 0.015 and $3 \times 10^{-3}$ for the Legacy and Generic workflows, respectively) given the Generic template.

With respect to data break-up by contrast (fig. 3a, right panel), we see significant effects for the contrast $\left(F_{2,190}=4.026, p=.019\right)$, and the processing-contrast interaction $\left(F_{2,190}=17.05, p=1.55 \times 10^{-7}\right)$ factors. Multiple-comparison corrected post-hoc t-tests reveal that the processing factor remains significant in interaction with the T2w $+\operatorname{BOLD}(p=$ $.025), \mathrm{T} 2 \mathrm{w}+\mathrm{CBV}\left(p=1 \times 10^{-19}\right)$, and T1w $+\operatorname{BOLD}\left(p=9 \times 10^{-12}\right)$ of the contrast factor.

\subsection{Smoothness conservation}

A further aspect of preprocessing quality is the resulting image smoothness. Although controlled smoothing is a valuable tool used to increase the signal-to-noise ratio (SNR), uncontrolled smoothing limits operator discretion in the trade-off between SNR and feature granularity, leading to undocumented loss of spatial resolution and thus inferior anatomical alignment Esteban et al. (2019). To ascertain the extent of uncontrolled smoothing, we compute a Smoothness Conservation Factor (SCF), expressing the ratio between the smoothness of the preprocessed images and the smoothness of the original images. Similarly to the VCF metric, perfect smoothness conservation is given by $\mathrm{SCF}=1$, though deviations are unavoidalbe due to interpolation and are further impacted by volume effect.

With respect to the data shown in fig. 3b (left panel), we note that SCF is sensitive to the template $\left(F_{1,388}=69.48, p=1.35 \times 10^{-15}\right)$, the workflow $\left(F_{1,388}=260.2, p=3.64 \times 10^{-45}\right)$, but not the interaction of the 


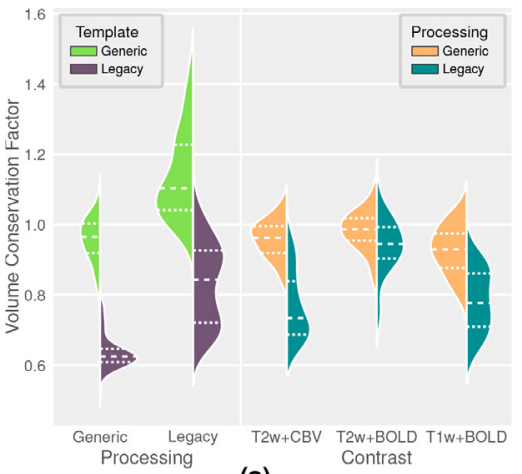

(a)

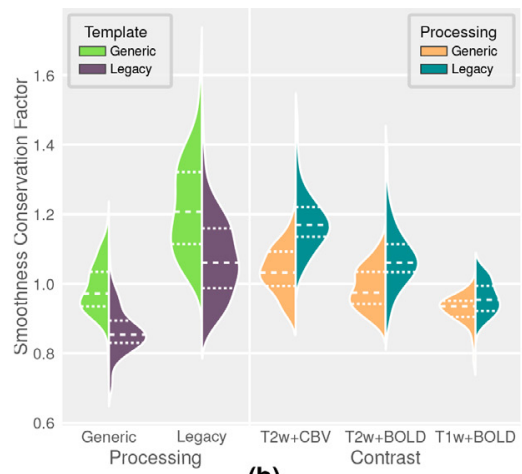

(b)

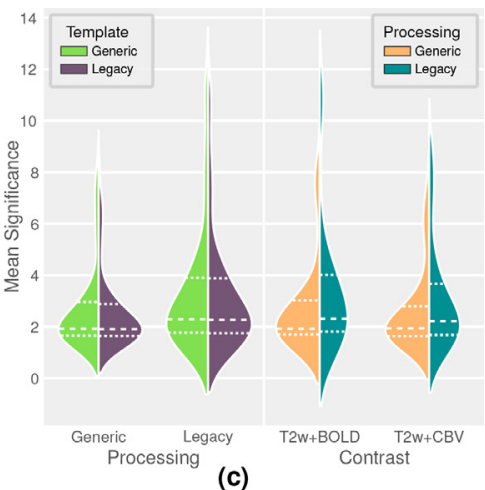

(c)

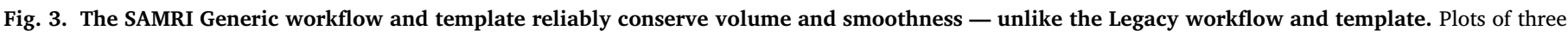

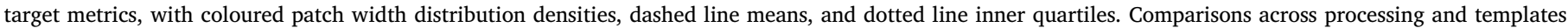
include all contrast combinations, comparisons across processing and contrasts include only matching template-workflow combinations.

factors $\left(F_{1,388}=1.365, p=.24\right)$. The performance of the Generic SAMRI workflow in conjunction with the Generic template is significantly different from that of the Legacy workflow in conjunction with the Legacy template, yielding a two-tailed $\mathrm{p}$-value of $2.7 \times 10^{-19}$. In this comparison, the root mean squared error ratio favours the Generic workflow $\left(\mathrm{RMSE}_{\mathrm{L}} / \mathrm{RMSE}_{\mathrm{G}} \simeq 1.9\right.$ ).

Descriptively, we observe that the Legacy level of the template variable introduces a smoothness reduction (SCF of $-0.12,95 \%$ CI : -0.14 to 0.10 ), while the Legacy level of the workflow variable introduces a smoothness gain (SCF of $0.24,95 \% \mathrm{CI}: 0.22$ to 0.25 ). Further, we note that there is a strong variance increase for the Legacy workflow - 3.08fold (variances of 0.014 and 0.0045 for the Legacy and Generic workflows, respectively) given the Legacy template, and 3.46-fold (variances of 0.018 and 0.0053 for the Legacy and Generic workflows, respectively) given the Generic template.

Given the break-up by contrast shown in fig. 3b (right panel), we see significant effects for the contrast factor $\left(F_{2,190}=21.55, p=3.68 \times\right.$ $\left.10^{-9}\right)$ and the processing-contrast interaction $\left(F_{2,190}=9.576, p=.00011\right)$. Multiple-comparison corrected post-hoc t-tests reveal that the processing factor remains significant in interaction with the T2 $\mathrm{w}+\operatorname{BOLD}(p=$ $\left.1 \times 10^{-6}\right), \mathrm{T} 2 \mathrm{w}+\mathrm{CBV}\left(p=2 \times 10^{-14}\right)$ and $\mathrm{T} 1 \mathrm{w}+\operatorname{BOLD}(p=.046)$ of the contrast factor.

\subsection{Functional analysis}

Functional analysis is a frequently used avenue for preprocessing QC, and has some viability as it is a feature not weighted by the registration process. However, the method is computationally intensive and only applicable to stimulus-evoked functional data. Additionally, functional analysis significance scores are sensitive to data smoothness Molloy et al. (2014), and thus an increased score on account of uncontrolled smoothing can be expected. For this analysis we compute the Mean Significance (MS), expressing the significance detected across all voxels of a scan, for the subset of contrasts which feature stimulusevoked activity (T2w + CBV and T2w + BOLD).

As seen in fig. 3c (left panel), MS is sensitive to the workflow $\left(F_{1,268}=\right.$ $4.001, p=.046)$, but not to the template $\left(F_{1,268}=0.054, p=.82\right)$, or the interaction of the factors $\left(F_{1,268}=1.01 \times 10^{-6}, p=1\right)$.

The performance of the SAMRI Generic workflow (with the Generic template) differs significantly from that of the Legacy workflow (with the Legacy template) in terms of MS, yielding a two-tailed p-value of $2.8 \times 10^{-9}$.

Descriptively, we observe that the Legacy level of the workflow variable introduces a notable significance increase (MS of $0.70,95 \% \mathrm{CI}$ : 0.53to0.87), while the Legacy level of the template variable (MS of $-0.08,95 \% \mathrm{CI}:-0.25$ to 0.09 ), and the interaction of the Legacy template and Legacy workflow (MS of 0.00, 95\%CI : -0.24to0.24) introduce no significance change. Furthermore, we again note a variance increase in all applications of the Legacy workflow Further, we note that there is a strong variance increase for the Legacy workflow -2.45 -fold (variances of 5.51 and 2.25 for the Legacy and Generic workflows, respectively) given the Legacy template, and 2.26-fold ((variances of 6.07 and 2.69 for the Legacy and Generic workflows, respectively) given the Generic template.

With respect to data break-up by contrast (fig. 3c, right panel), we see no notable main effect for the contrast variable $\left(F_{1,132}=0.03, p=.86\right)$, nor the contrast-template interaction $\left(F_{1,132}=0.091, p=.76\right)$.

Functional analysis effects can further be inspected by visualizing population-level maps. Second-level t-statistic maps depicting the $\mathrm{T} 2 \mathrm{w}+\mathrm{CBV}$ and $\mathrm{T} 2 \mathrm{w}+\mathrm{BOLD}$ omnibus signal (common to all subjects and sessions) provide a succinct overview capturing signal amplitude, directionality, and coverage (fig. 4). We note that the Legacy workflow induces coverage overflow (figs. $4 \mathrm{~d}, 4 \mathrm{~g}$ and $4 \mathrm{~h}$ ), while the Legacy template causes acquisition slice misalignment (figs. $4 \mathrm{~b}, 4 \mathrm{~d}, 4 \mathrm{f}$ and $4 \mathrm{~h}$ ). Positive activation of the Raphe system, most clearly disambiguated from the surrounding tissue in the T2w + BOLD contrast, is notably displaced very far caudally by the joint effects of the Legacy workflow and the Legacy template (fig. 4h). Processing with the Generic template and workflow (figs. 4a, 4 e) successfully mitigates all of these issues.

\subsection{Variance analysis}

An additional assessment of preprocessing quality focuses on the robustness to longitudinal data variability, and whether this is attained without overfitting (i.e. compromising physiologically meaningful variability). The core assumption of this analysis is that registration should be less susceptible to experiment repetition and aging over an 8-week period in adult mice, than to inter-individual differences. Consequently, similarity scores of preprocessed scans with respect to the target template should show more variation across subject variable levels than session variable levels. This comparison can be performed using a type 3 ANOVA of a general linear model fit including a subject and a session level, and with heteroscedasticity-consistent covariance estimators MacKinnon and White, 1985.

For this assessment we select three metrics: Neighborhood Cross Correlation (CC, sensitive to localized correlation), Global Correlation (GC, sensitive to whole-image correlation), and Mutual Information (MI, sensitive to whole-image information similarity). For this comparison we select animals in the mature and middle aged brackets (3 to 14 months) Flurkey et al. (2007), within which inter-session variability is more clearly distinguished as physiologically less meaningful than inter-subject variability. We further target this descriptive statistical 


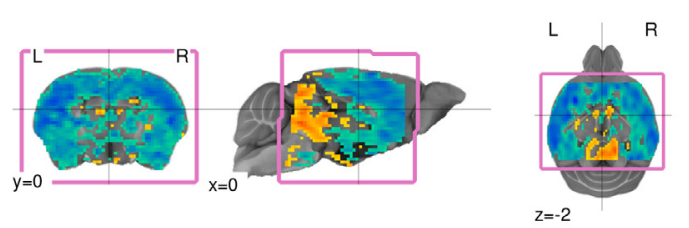

(a) Generic workflow with Generic template T2w+CBV map: correct slice orientation and coverage bounds.
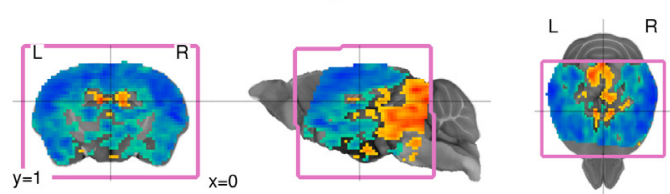

$\mathrm{z}=7$

(c) Legacy workflow with Generic template T2w+CBV map: distorted slice orientation and correct coverage bounds.
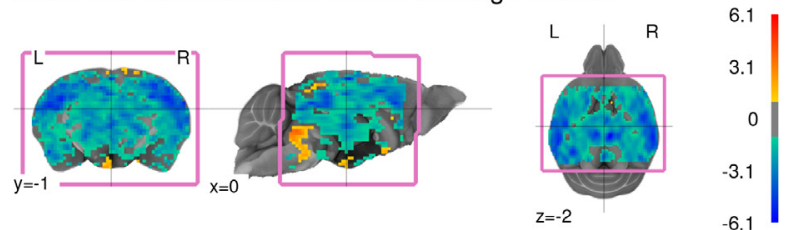

(e) Generic workflow with Generic template T2w+BOLD map: correct slice orientation and coverage bounds.
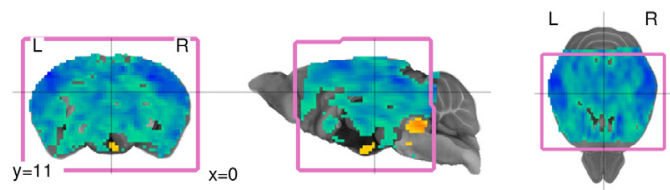

$z=8$

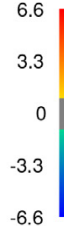

(g) Legacy workflow with Generic template T2w+BOLD map: correct slice orientation and overflowing coverage.

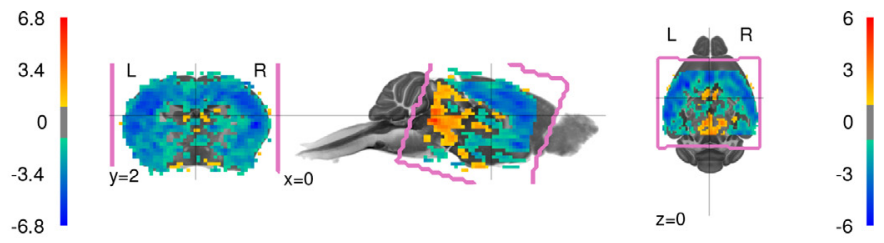

(b) Generic workflow with Legacy template T2w+CBV map: distorted slice orientation and correct coverage bounds.
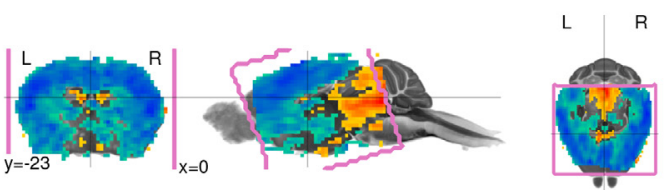

$z=6$

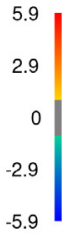

(d) Legacy workflow with Legacy template T2w+CBV map: distorted slice orientation and overflowing bounds.
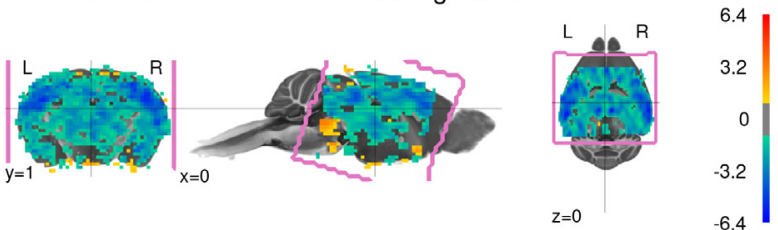

(f) Generic workflow with Legacy template T2w+BOLD map: distorted slice orientation and correct coverage bounds.
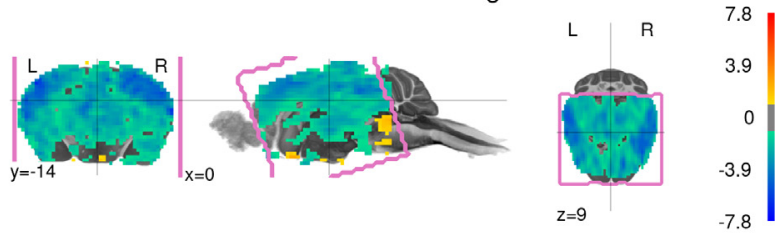

(h) Legacy workflow with Legacy template T2w+BOLD map: distorted slice orientation and overflowing coverage.

Fig. 4. Generic processing eliminates map overflow into adjacent anatomical regions, and Generic template usage reduces slice orientation distortions. As seen in multiplanar depictions of second-level omnibus statistic maps, thresholded at $|t| \geq 1$. The acquisition area is bracketed in pink, and comparison with statistic coverage should account for the latter being more constrained, as the omnibus statistic contrast is only defined for voxels captured in every evaluated scan.
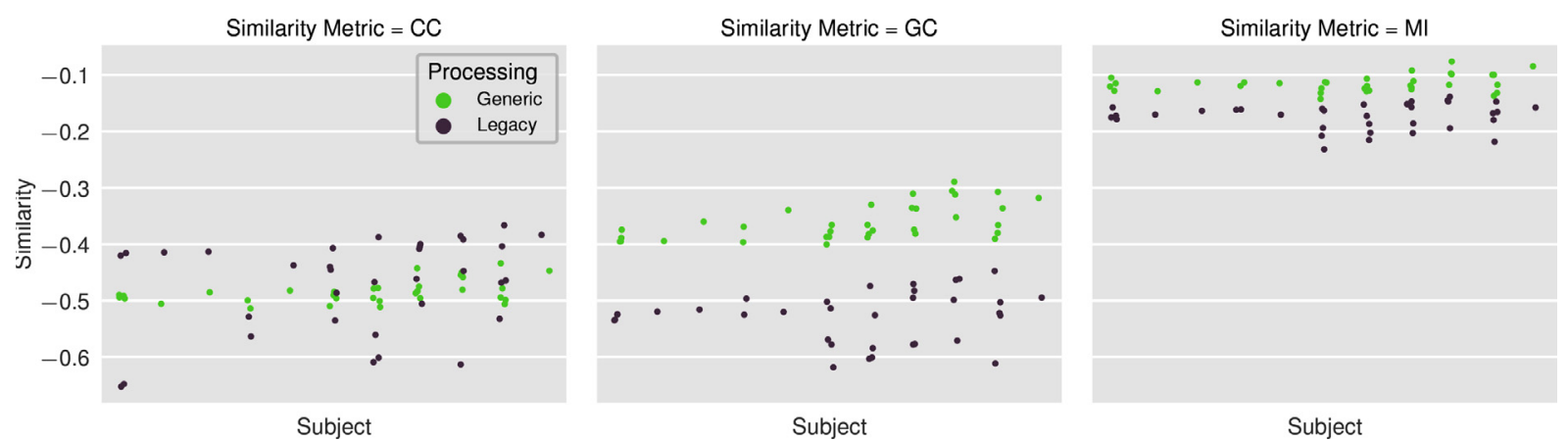

Fig. 5. The SAMRI Generic workflow conserves subject-wise variability and minimizes trial-to-trial variability compared to the Legacy workflow. Swarmplots illustrate similarity metric scores between preprocessed images and the corresponding workflow template, plotted across subjects (x-axis bins) and sessions (individual points in each $\mathrm{x}$-axis bin), for the CBV contrast.

analysis which is based on the distribution and therefore ample presence of variance, at the matching workflow-template comparison for the $\mathrm{T} 2 \mathrm{w}+\mathrm{CBV}$ contrast, which shows the strongest effect in both VCF and SCF.

fig. 5 renders similarity scores for the SAMRI Generic and Legacy workflows (considering only the matching workflow-template combinations). The SAMRI Legacy workflow and template combination produces results with higher F-statistics for the session than for the subject variable, across all metrics: CC (subject: $F_{10,19}=2.881, p=.023$, session: $F_{4,19}=3.498, p=.027$ ), GC (subject: $F_{10,19}=0.72, p=.7$, session: $F_{4,19}=3.271, p=.034$ ), and MI (subject: $F_{10,19}=0.81, p=.63$, session: $\left.F_{4,19}=2.985, p=.045\right)$.
For the SAMRI Generic workflow this pattern reverses in 2 of the 3 assessed metrics, with overall higher F-statistic scores for the subject variable than for the session variable: CC (subject: $F_{10,19}=7.845, p=7.04 \times$ $10^{-5}$, session: $F_{4,19}=2.617, p=.068$ ), GC (subject: $F_{10,19}=2.799, p=$ .026 , session: $F_{4,19}=1.312, p=.3$ ), and MI (subject: $F_{10,19}=7.812, p=$ $7.25 \times 10^{-5}$, session: $F_{4,19}=1.956, p=.14$ ).

\section{Discussion}

The workflow and template design presented herein represent a significant methodological improvement in preclinical MRI, with both prospective and retroactive applicability, in conjunction with BIDS data 
or vendor data and vendor-to-BIDS Ioanas et al., 2020 conversion workflows. The workflow offers significant advantages by reducing coverage overestimation and uncontrolled smoothing, and by improving sessionto-session consistency. This is most prominently highlighted by Volume Conservation (fig. 3a), Smoothness Conservation (fig. 3b), and Variance Analysis (fig. 5), where the combined usage of the SAMRI Generic workflow and template outperforms all other combinations of the multifactorial analysis. Increased region assignment validity is also revealed in a qualitative examination of higher-level functional maps (fig. 4), where only the Generic workflow and template combination provides accurate coverage of the sampled volume for stimulus-evoked fMRI data.

These benefits are robust with respect to tested contrast combinations (fig. 3), with the Generic workflow-template combination being less or equally susceptible to the contrast variable compared to the Legacy workflow-template combination. The performance of the Generic workflow is more consistent across all metrics, as demonstrated by notable standard deviation reductions for VCS, SCF, and MS. Variance analysis reveals that the Generic workflow in conjunction with the Generic template improves registration robustness - with respect to session-specific preparation and positioning, as well as adult animal age progression over a period of 8 weeks - and better conserves subjectspecific features, as compared to the Legacy workflow and template combination.

The Benchmarking data collection, which includes animals with optogenetic implants, showcases workflow robustness with respect to both magnetic susceptibility artefacts (arising from scar tissue around the dental cement attachment), as well as volumetric distortion resulting from brain lesion and implant presence. Further, this last feature reveals reliable multi-session implant position conservation for the Generic (fig. S3) but not the Legacy (fig. S4) workflow. Robustness to further sources of data variability is substantiated by SNR heterogeneity in the benchmarking data collection, which is acquired not only with different scan protocols and with or without a contrast agent, but also with different coils (in-house T/R surface coil for T2w + BOLD and T2w + CBV, and cryogenic coil for T1 $w+$ BOLD). In fact, the surface coil protocol constitutes a challenging and unfavourable setting for registration due to its intrinsic $B_{1}$ gradient. In spite of this, registration remains robust. Lastly, the registration pipeline contains a bias field correction step which is optimized to provide significantly more aggressive behaviour than the default parameters - with respect to both its spatial frequency threshold and amplitude gain. This renders further homogeneity variations (in the spatial resolution range obtained by using different field strengths or resonator coils) mathematically equivalent to SNR variations.

Closer model inspection reveals that in addition to the processing factor, the template factor also drives variability. The Legacy template induces both volume and smoothness decrease below raw data values (fig. 3a, 3 b). This clearly indicates a whole-volume effect, whereby a target template smaller than the recoded brain causes contraction during registration, affecting both volume and smoothness. We thus highlight the importance of an appropriate template choice, and strongly recommend usage of the Generic template on account of better scale similarity to data acquired in adult mice.

The volume conservation, smoothness conservation, and session-tosession consistency of the SAMRI Generic workflow and template combination are further complemented by numerous design benefits (figs. 1, 2). These include increased transparency and parameterization (easing inspection and further customization), veracity of resulting data headers, and spatial coordinates more meaningful for surgical interventions.

\subsection{Quality control}

A major contribution of this work is the implementation of multiple metrics providing simple and thorough $\mathrm{QC}$ for registration performance (VCF, SCF, MS, and Variance Analysis); and the release of a data collection Ioanas and Rudin (2019); Zerbi and Wenderoth (2020) suitable for multifactorial benchmarking.
The VCF and SCF provide good quantitative estimates of distortion prevalence. The analysis comparing subject-wise and session-wise variance is a conceptually interesting avenue for ascertaining how much a registration workflow is potentially overfitting. It should, however, primarily be understood as a descriptive statistical metric and re-used with caution, as it is highly sensitive to heteroscedasticity and whether or not the covariance estimator appropriately accounts for it. These metrics are relevant to both preclinical and clinical MRI workflow improvements.

Global statistical power is not a reliable metric for registration optimization. Regrettably, however, it may be the most prevalently used if results are only inspected at a higher level — and could bias analysis. This is exemplified by the positive main effect of the Legacy workflow seen in fig. 3c. In this particular case, optimizing for statistical power alone would give a misleading indication, as becomes evident when all metrics are inspected.

We suggest that a VCF, SCF and Variance based comparison, coupled with visual inspection of a small number of omnibus statistic maps is a feasible and sufficient tool for benchmarking workflows. We recommend reuse of the presented data for workflow benchmarking, as they include (a) multiple sources of variation (contrast, session, subjects), (b) functional activity with broad coverage but spatially distinct features, and (c) significant distortions due to implant properties — which are appropriate for testing workflow robustness. In addition to the workflow code Ioanas et al. (2017a), we openly release the re-executable source code Ioanas et al. (2019a) for all statistics and figures in this document. Thus both the novel method and the benchmarking process are fully transparent and reusable with further data.

\subsection{Conclusion}

We present a novel registration workflow, "SAMRI Generic", which offers several advantages compared to ad hoc approaches commonly used for small animal MRI. In-depth multivariate comparison with a thoroughly documented Legacy pipeline revealed superior performance of the SAMRI Generic workflow in terms of volume and smoothness conservation, as well as variance structure across subjects and sessions. The metrics introduced for registration $\mathrm{QC}$ are not restricted to the processing of small animal fMRI data, and can be readily expanded to other brain imaging applications. The optimized registration parameters of the SAMRI Generic Workflow are easily accessible in the source code and transferable to any other workflows making use of the ANTs package. Overall, we believe that the SAMRI Generic workflow should facilitate and harmonize processing of mouse brain imaging data across studies and centers.

\section{Methods}

\subsection{Data}

For the quality control of the workflows, a dataset with an effective size of 164 scans is used. Scans were acquired using a Bruker PharmaScan system (7T, $16 \mathrm{~cm}$ bore), and variable coils. Experimental procedures were approved by the Veterinary Office of the Canton of Zurich in accordance with relevant regulations.

All animals were anesthetized for scanning with medetomidine $(0.05 \mathrm{mg} / \mathrm{kg} \mathrm{BW}$ bolus and $0.1 \mathrm{mg} / \mathrm{kg}$ BWh intraperitoneal infusion) and isoflurane $0.5 \%$, the protocol being derived from the previously published "Med/Iso" specification Grandjean et al. (2014).

\subsection{1. $T 2 w+C B V$ And $T 2 w+B O L D$}

Data was acquired from 11 adult ePet-Cre Scott et al. (2005) C57BL/6 mice (6 males and 5 females, 207 to 261 days old at study onset), with each animal scanned on up to 5 sessions, at 14 day intervals. Each session contains an anatomical scan and two functional scans - with Blood-Oxygen Level Dependent (BOLD) Ogawa et al. (1990) and Cerebral Blood Volume (CBV) 
Marota et al. (1999) contrast, respectively (for a total of 68 functional scans).

Anatomical scans were acquired via a TurboRARE sequence, with a RARE factor of 8 , an echo time (TE) of $21 \mathrm{~ms}$, an inter-echo spacing of $7 \mathrm{~ms}$, and a repetition time (TR) of $2500 \mathrm{~ms}$, sampled sagittally at $\Delta \mathrm{x}(\nu)=166.7 \mu \mathrm{m}$, horizontally at $\Delta \mathrm{y}(\phi)=75 \mu \mathrm{m}$, and coronally at $\Delta z(t)=650 \mu \mathrm{m}$ (slice thickness of $500 \mu \mathrm{m}$ ). The field of view covered $20 \times 9 \mathrm{~mm}^{2}$ and was sampled via a $120 \times 120$ matrix. A total of 14 slices were acquired.

The functional BOLD and CBV scans were acquired using a gradientecho Echo Planar Imaging (ge-EPI) sequence with a flip angle of $60^{\circ}$ and with $\mathrm{TR} / \mathrm{TE}=1000 \mathrm{~ms} / 15 \mathrm{~ms}$ and $\mathrm{TR} / \mathrm{TE}=1000 \mathrm{~ms} / 5.5 \mathrm{~ms}$, respectively. Data were sampled at $\Delta x(\nu)=312.5 \mu \mathrm{m}, \Delta \mathrm{y}(\phi)=281.25 \mu \mathrm{m}$, and $\Delta \mathrm{z}(\mathrm{t})=650 \mu \mathrm{m}$ (slice thickness of $500 \mu \mathrm{m}$ ). The field of view covered 20 $\times 9 \mathrm{~mm}^{2}$ and was sampled via a $64 \times 32$ matrix. A total of 14 slices were acquired.

Animals were fitted with an optic fiber implant $(1=3.2 \mathrm{~mm} \mathrm{~d}=400 \mu \mathrm{m})$ targeting the Dorsal Raphe (DR) nucleus 14 days or longer prior to the first session. The nucleus was sensitized to optical stimulation by transgenic expression of Cre recombinase under the ePet promoter Scott et al. (2005) and rAAV delivery of a plasmid for Cre-conditional Channelrhodopsin and YFP expression - pAAV-EF1a-double floxed-hChR2(H134R)-EYFP-WPRE-HGHpA, reposited by Karl Deisseroth (Addgene plasmid \#20298). Scans were acquired using an in-house T/R surface coil, engineered to permit optic fiber protrusion.

The DR was stimulated via an Omicron LuxX 488-60 laser (488nm) tuned to $30 \mathrm{~mW}$ at contact with the fiber implant, according to the protocol listed in table S1. Stimulation was delivered and reports recorded via the COSplay system Aymanns et al. (2019).

\subsection{2. $T 1 w+B O L D$}

Data was acquired from 10 adult male C57BL/6 mice (Jackson laboratory, Maine, USA), which were 34 days old at study onset, with each animal scanned on 3 sessions (repeated at 58 days postnatal and 112 days postnatal) Zerbi et al., 2018. Each session contains an anatomical scan and a functional BOLD contrast scan (for a total of 30 functional scans).

Anatomical scans were acquired via a Fast low-angle shot (FLASH) sequence with a RARE factor of 1 , a flip angle of $30^{\circ}$, and $\mathrm{TR} / \mathrm{TE}=522 \mathrm{~ms} / 3.51 \mathrm{~ms}$. Data were sampled at $\Delta \mathrm{x}(\nu)=52 \mu \mathrm{m}$, $\Delta \mathrm{y}(\phi)=26 \mu \mathrm{m}, \Delta \mathrm{z}(\mathrm{t})=500 \mu \mathrm{m}$ (slice thickness of $500 \mu \mathrm{m}$ ). The field of view covered $20 \times 10 \mathrm{~mm}^{2}$ and was sampled via a $384 \times 384$ matrix. A total of 20 slices were acquired.

The functional BOLD scans were acquired using a gradient-echo Echo Planar Imaging (ge-EPI) sequence with a flip angle of $60^{\circ}$ and $\mathrm{TR} / \mathrm{TE}=1000 \mathrm{~ms} / 15 \mathrm{~ms}$. Data were sampled at $\Delta \mathrm{x}(\nu)=222 \mu \mathrm{m}$, $\Delta \mathrm{y}(\phi)=200 \mu \mathrm{m}$, and $\Delta \mathrm{z}(\mathrm{t})=500 \mu \mathrm{m}$ (slice thickness of $500 \mu \mathrm{m}$ ). The field of view covered $20 \times 10 \mathrm{~mm}^{2}$ and was sampled via a $90 \times 50$ matrix. A total of 20 slices were acquired.

\subsection{Metrics}

For the VCF we use the $66^{\text {th }}$ intensity percentile of the raw scan as a signal threshold — as determined by operator inspection to best approximate brain selection by signal intensity, without over-optimization. It should be noted that the percentile threshold neither can nor needs to select brain voxels exclusively, as the transformation is performed on unmasked data (lower thresholds will simply weigh the metric towards additional non-brain structure conservation, and higher thresholds will simply constrain the amount of brain tissue sampled for comparison). The arbitrary unit equivalent of this threshold is recorded per-scan and applied to all preprocessing results for that particular scan - eq. (1), where $v$ is the voxel volume in the original space, $v^{\prime}$ the voxel volume in the transformed space, $n$ the number of voxels in the original space, $m$ the number of voxels in the transformed space, $s$ a voxel value sampled from the vector $S$ containing all values in the original data, and $s^{\prime}$ a voxel value sampled from the transformed data.

$V C F=\frac{v^{\prime} \sum_{i=1}^{m}\left[s_{i}^{\prime} \geq P_{66}(S)\right]}{v \sum_{i=1}^{n}\left[s_{i} \geq P_{66}(S)\right]}=\frac{v^{\prime} \sum_{i=1}^{m}\left[s_{i}^{\prime} \geq P_{66}(S)\right]}{v\lceil 0.66 n\rceil}$

The SCF metric measures the pre- and post-processing smoothness ratio, defined as the full-width at half-maximum (FWHM) of the signal amplitude spatial autocorrelation function (ACF Eklund et al. (2016)). As fMRI data have non-Gaussian spatial ACF, we use AFNI Cox (1996) to fit the following function - eq. (2), where $r$ is the distance of two amplitude distribution samples, $a$ is the relative weight of the Gaussian, $b$ is the width of the Gaussian, and $c$ the decay of the mono-exponential term Cox et al. (2017).

$A C F(r)=a * e^{-r^{2} /\left(2 * b^{2}\right)}+(1-a)+e^{-r / c}$

Statistical power as per the MS metric is represented by the negative logarithm of first-level p-values. This produces voxelwise statistical estimates for the probability that a time course could - by chance alone - be at least as well correlated with the stimulation regressor as the voxel time course measured. We compute the per-scan average of these values as seen in eq. (3), where $n$ represents the number of statistical estimates in the scan, and $p$ is a p-value.

$M S=\frac{\sum_{i=1}^{n}-\log \left(p_{i}\right)}{n}$

Two statistical models, including main effects and interaction terms, were evaluated for each metric. The first details workflow and template effects, and the second details contrast effects. Multiple comparison correction was performed via the Benjamini-Hochberg method, fixing the false discovery rate (FDR) $\alpha$ at 0.05 .

\subsection{Software}

The workflow functions leverage the Nipype Gorgolewski et al. (2011), FSL Jenkinson et al. (2012) and ANTs Avants et al. (2011) packages. Software management relevant for verifying the reproducibility of the software environment and analysis results was performed via neuroscience package install instructions for the Gentoo Linux distribution Ioanas et al., 2017. For Quality Control we distribute as part of this article workflows using the SciPy Millman and Aivazis (2011), pandas McKinney (2010), Seaborn Waskom et al. (2017), and Statsmodels Seabold and Perktold (2010) packages.

\section{Reproducibility and resource availability}

All data is freely available Ioanas and Rudin (2019); Zerbi and Wenderoth (2020), and automatically accessible via the Gentoo Linux package manager. All code relevant for reproducing this document is freely available Ioanas et al. (2019a), and compliant with the RepSeP specification Ioanas, Rudin, 2018 to allow article reproduction based solely on the raw data and top-level code of the article.

\section{Credit authorship contribution statement}

Horea-Ioan Ioanas: Conceptualization, Methodology, Software, Validation, Formal analysis, Investigation, Data curation, Writing original draft, Writing - review \& editing, Visualization, Supervision, Project administration. Markus Marks: Methodology, Software, Validation, Formal analysis, Investigation, Writing - original draft, Writing - review \& editing, Visualization. Valerio Zerbi: Resources, Data curation, Writing - original draft. Mehmet Fatih Yanik: Writing - original draft, Supervision. Markus Rudin: Writing - original draft, Writing review \& editing, Supervision, Funding acquisition. 


\section{References}

Anderson, R.J., Cook, J.J., Delpratt, N., Nouls, J.C., Gu, B., McNamara, J.O., Avants, B.B., Johnson, G.A., Badea, A., 2019. Small animal multivariate brain analysis (samba)a high throughput pipeline with a validation framework. Neuroinformatics 17 (3), 451-472. doi:10.1007/s12021-018-9410-0.

Avants, B.B., Tustison, N.J., Song, G., Cook, P.A., Klein, A., Gee, J.C., 2011. A reproducible evaluation of ANTs similarity metric performance in brain image registration. Neuroimage 54 (3), 2033-2044. doi:10.1016/j.neuroimage.2010.09.025.

Aymanns, F., Rudin, M., Ioanas, H.-I., 2019. COSplay: Contrast optimized stimulation player. Journal of Open Source Software 4 (39), 1171. doi:10.21105/joss.01171.

Carp, J., 2012. The secret lives of experiments: methods reporting in the fmri literature. Neuroimage 63 (1), 289-300. doi:10.1016/j.neuroimage.2012.07.004

Cox, R.W., 1996. AFNI: Software for analysis and visualization of functional magnetic resonance neuroimages. Comput. Biomed. Res. 29 (3), 162-173. doi:10.1006/cbmr.1996.0014.

Cox, R.W., Ashburner, J., Breman, H., Fissell, K., Haselgrove, C., Holmes, C.J., Lancaster, J.L., Rex, D.E., Smith, S.M., Woodward, J.B., Strother, S.C., 2004. A (Sort of) New Image Data Format Standard: NIfTI-1. OHBM.

Cox, R.W., Chen, G., Glen, D.R., Reynolds, R.C., Taylor, P.A., 2017. FMRI Clustering in AFNI: false-positive rates redux. Brain Connect 7 (3), 152-171. doi:10.1089/brain.2016.0475

Dorr, A., Lerch, J., Spring, S., Kabani, N., Henkelman, R., 2008. High resolution threedimensional brain atlas using an average magnetic resonance image of 40 adult c57bl/6j mice. Neuroimage 42 (1), 60-69. doi:10.1016/j.neuroimage.2008.03.037.

Eklund, A., Nichols, T.E., Knutsson, H., 2016. Cluster failure: why fMRI inferences for spatial extent have inflated false-positive rates. Proceedings of the National Academy of Sciences 201602413. doi:10.1073/pnas.1602413113.

Esteban, O., Markiewicz, C., Blair, R.W., Moodie, C., Isik, A.I., Aliaga, A.E., Kent, J., Goncalves, M., DuPre, E., Snyder, M., et al., 2019. FMRIPRep: a robust preprocessing pipeline for functional MRI. Nat. Methods 111116. doi:10.1038/s41592-018-0235-4.

Flurkey, K., Currer, J.M., Harrison, D., 2007. Mouse Models in Aging Research. In: The mouse in biomedical research. Elsevier, pp. 637-672.

Gorgolewski, K., Burns, C.D., Madison Cindeeand Clark, D., Halchenko, Y.O., Waskom, M.L., Ghosh, S.S., 2011. Nipype: a flexible, lightweight and extensible neuroimaging data processing framework in python. Front. Neuroinform. 5. doi:10.3389/fninf.2011.00013.

Gorgolewski, K.J., Auer, T., Calhoun, V.D., Craddock, R.C., Das, S., Duff, E.P., Flandin, G., Ghosh, S.S., Glatard, T., Halchenko, Y.O., et al., 2016. The brain imaging data structure, a format for organizing and describing outputs of neuroimaging experiments. Sci Data 3, 160044. doi:10.1038/sdata.2016.44.

Grandjean, J., Schroeter, A., Batata, I., Rudin, M., 2014. Optimization of anesthesia protocol for resting-state fmri in mice based on differential effects of anesthetics on functional connectivity patterns.. Neuroimage 102 Pt 2, 838-847. doi:10.1016/j.neuroimage.2014.08.043.

Ioanas, H.-I., Marks, M., Schmidt, D., Aymanns, F., Rudin, M., 2017a. SAMRI — Small Animal Magnetic Resonance Imaging. 10.5281/zenodo.1044033

Ioanas, H.-I., Marks, M., Yanik, M. F., Rudin, M., 2019a. Source Code for "An Optimized Registration Workflow and Standard Geometric Space for Small Animal Brain Imaging". 10.5281/zenodo.2650431

Ioanas, H.-I., Marks, M., Garin, C.M., Dhenain, M., Yanik, M.F., Rudin, M., 2020. An automated open-source workflow for standards-compliant integration of small animal magnetic resonance imaging data. Front Neuroinform 14, 5. doi:10.3389/fninf.2020.00005.

Ioanas, H.-I., Rudin, M., 2019. BIDS Data for "An Optimized Registration Workflow and Standard Geometric Space for Small Animal Brain Imaging". 10.5281/zenodo. 3445428

Ioanas, H.-I., Segessemann, T., Rudin, M., 2019b. "Mouse Brain Atlases" generator workflows. 10.5281/zenodo. 2545838

Ioanas, H.-I., Rudin, M., 2018. Reproducible self-publishing for Python-based research. EuroSciPy doi:10.6084/m9.figshare.7247339.v1.

Ioanas, H.-I., Saab, B., Rudin, M., 2017. Gentoo linux for neuroscience-a replicable, flexible, scalable, rolling-release environment that provides direct access to development software. Research Ideas and Outcomes 3, e12095. doi:10.3897/rio.3.e12095.
Janke, A.L., Ullmann, J.F., 2015. Robust methods to create ex vivo minimum deformation atlases for brain mapping. Methods 73, 18-26. doi:10.1016/j.ymeth.2015.01.005.

Jenkinson, M., Beckmann, C.F., Behrens, T.E.J., Woolrich, M.W., Smith, S.M., 2012. FSL. Neuroimage 62 (2), 782-790. doi:10.1016/j.neuroimage.2011.09.015.

Lein, E.S., Hawrylycz, M.J., Ao, N., Ayres, M., Bensinger, A., Bernard, A., Boe, A.F., Boguski, M.S., Brockway, K.S., Byrnes, E.J., Chen, L., Chen, L., Chen, T.-M., Chin, M.C., Chong, J., Crook, B.E., Czaplinska, A., Dang, C.N., Datta, S., Dee, N.R., Desaki, A.L., Desta, T., Diep, E., Dolbeare, T.A., Donelan, M.J., Dong, H.-W., Dougherty, J.G., Duncan, B.J., Ebbert, A.J., Eichele, G., Estin, L.K., Faber, C., Facer, B.A., Fields, R., Fischer, S.R., Fliss, T.P., Frensley, C., Gates, S.N., Glattfelder, K.J., Halverson, K.R., Hart, M.R., Hohmann, J.G., Howell, M.P., Jeung, D.P., Johnson, R.A., Karr, P.T., Kawal, R., Kidney, J.M., Knapik, R.H., Kuan, C.L., Lake, J.H., Laramee, A.R., Larsen, K.D., Lau, C., Lemon, T.A., Liang, A.J., Liu, Y., Luong, L.T., Michaels, J., Morgan, J.J., Morgan, R.J., Mortrud, M.T., Mosqueda, N.F., Ng, L.L., Ng, R., Orta, G.J., Overly, C.C., Pak, T.H., Parry, S.E., Pathak, S.D., Pearson, O.C., Puchalski, R.B., Riley, Z.L., Rockett, H.R., Rowland, S.A., Royall, J.J., Ruiz, M.J., Sarno, N.R., Schaffnit, K., Shapovalova, N.V., Sivisay, T., Slaughterbeck, C.R., Smith, S.C., Smith, K.A., Smith, B.I., Sodt, A.J., Stewart, N.N., Stumpf, K.-R., Sunkin, S.M., Sutram, M., Tam, A., Teemer, C.D., Thaller, C., Thompson, C.L., Varnam, L.R., Visel, A., Whitlock, R.M., Wohnoutka, P.E., Wolkey, C.K., Wong, V.Y., Wood, M., Yaylaoglu, M.B., Young, R.C., Youngstrom, B.L., Yuan, X.F., Zhang, B., Zwingman, T.A., Jones, A.R., 2006. Genome-wide atlas of gene expression in the adult mouse brain. Nature 445 (7124), 168-176. doi:10.1038/nature05453.

MacKinnon, J.G., White, H., 1985. Some heteroskedasticity-consistent covariance matrix estimators with improved finite sample properties. J Econom 29 (3), 305-325. doi:10.1016/0304-4076(85)90158-7.

Marota, J.J., Ayata, C., Moskowitz, M.A., Weisskoff, R.M., Rosen, B.R., Mandeville, J.B., 1999. Investigation of the early response to rat forepaw stimulation. Magn Reson Med 41 (2), 247-252. doi:10.1002/(sici)1522-2594(199902)41:2<247::aid-mrm6 > 3.0.co;2-u.

McKinney, W., 2010. Data Structures for Statistical Computing in Python. In: van der Walt, S., Millman, J. (Eds.), Proceedings of the 9th Python in Science Conference, pp. 51-56.

Millman, K.J., Aivazis, M., 2011. Python for scientists and engineers. Computing in Science \& Engineering 13 (2), 9-12. doi:10.1109/mcse.2011.36.

Molloy, E.K., Meyerand, M.E., Birn, R.M., 2014. The influence of spatial resolution and smoothing on the detectability of resting-state and task fmri. Neuroimage 86, 221230. doi:10.1016/j.neuroimage.2013.09.001.

Ogawa, S., Lee, T.-M., Nayak, A.S., Glynn, P., 1990. Oxygenation-sensitive contrast in magnetic resonance image of rodent brain at high magnetic fields. Magn Reson Med 14 (1), 68-78. doi:10.1002/mrm.1910140108.

Oguz, I., Zhang, H., Rumple, A., Sonka, M., 2014. RATS: Rapid automatic tissue segmentation in rodent brain MRI. J. Neurosci. Methods 221, 175-182. doi:10.1016/j.jneumeth.2013.09.021.

Scott, M.M., Wylie, C.J., Lerch, J.K., Murphy, R., Lobur, K., Herlitze, S., Jiang, W., Conlon, R.A., Strowbridge, B.W., Deneris, E.S., 2005. A genetic approach to access serotonin neurons for in vivo and in vitro studies.. Proc. Natl. Acad. Sci. U.S.A. 102, 16472-16477. doi:10.1073/pnas.0504510102.

Seabold, S., Perktold, J., 2010. Statsmodels: Econometric and statistical modeling with Python. In: 9th Python in Science Conference.

Waskom, M., Botvinnik, O., O'Kane, D., Hobson, P., Lukauskas, S., Gemperline, D. C., Augspurger, T., Halchenko, Y., Cole, J. B., Warmenhoven, J., de Ruiter, J., Pye, C., Hoyer, S., Vanderplas, J., Villalba, S., Kunter, G., Quintero, E., Bachant, P., Martin, M., Meyer, K., Miles, A., Ram, Y., Yarkoni, T., Williams, M. L., Evans, C., Fitzgerald, C., Brian, Fonnesbeck, C., Lee, A., Qalieh, A., 2017. Seaborn: v0.8.1. 10.5281/zenodo.883859

Wood, T., Lythgoe, D., Williams, S., 2013. rBET: Making BET work for Rodent Brains. In: Proceedings of the ISMRM, p. 2706.

Zerbi, V., Ielacqua, G.D., Markicevic, M., Haberl, M.G., Ellisman, M.H., A-Bhaskaran, A., Frick, A., Rudin, M., Wenderoth, N., 2018. Dysfunctional autism risk genes cause circuit-specific connectivity deficits with distinct developmental trajectories. Cerebral Cortex 28 (7), 2495-2506. doi:10.1093/cercor/bhy046.

Zerbi, V., Wenderoth, N., 2020. BIDS wildtype data selection from "Dysfunctional Autism Risk Genes Cause Circuit-Specific Connectivity Deficits With Distinct Developmental Trajectories" doi:10.5281/zenodo.3885733. 